\title{
A Case of Dysphagia Aortica Caused by a Large Thoracic Aortic Aneurysm
}

\author{
Ha-Young Choi', Ung Jeon', Se-Whan Lee', Sang-Ho Park', Hye Yon Yu² \\ ${ }^{1}$ Department of Internal Medicine, Soonchunhyang University Cheonan Hospital; ${ }^{2}$ Department of Nursing, Soonchunhyang University College of Medicine, \\ Cheonan, Korea
}

\begin{abstract}
Dysphagia aortica is a rare condition defined by difficulty in swallowing and is caused by extrinsic compression of the esophagus due to an ectatic, tortuous, or aneurysmatic atherosclerotic thoracic aorta. It is easy to miss the diagnosis if the clinician does not consider the possibility of dysphagia caused by a thoracic aortic aneurysm. We present the case of an 82-year-old man who developed dysphagia aortica associated with a large thoracic aortic aneurysm. Extrinsic compression of the esophagus caused by an enlarged thoracic aorta was seen on upper gastrointestinal endoscopy, barium esophagogram, and chest computed tomography. With dietary modifications, his symptoms of dysphagia gradually improved.
\end{abstract}

Keywords: Deglutition disorders; Thoracic aortic aneurysm; Vascular compression

\section{INTRODUCTION}

Dysphagia is an alarming symptom that warrants prompt evaluation to identify the exact cause and initiate appropriate therapy. It might be due to a structural anomaly or abnormal motility in the passage of solids or liquids from the oral cavity to the stomach. Dysphagia aortica is a mechanical dysphagia due to the extrinsic compression of the esophagus by an ectatic, tortuous aorta or aneurysmal atherosclerotic aorta. The exact incidence of dysphagia aortica has not been established; however, it is rare [1]. We report here a case of an 82-year-old man with dysphagia aortica caused by a large thoracic aortic aneurysm (TAA).

\section{CASE REPORT}

An 82-year-old male patient was hospitalized for nausea and vomiting with dysphagia. He had a long-standing history of hypertension, chronic renal failure, and bronchiectasis. He was diagnosed with atrial fibrillation and heart failure 2 years ago and was treated with anticoagulants and diuretics. At that time, he complained of intermittent chest discomfort, and mediastinal widening was observed on chest X-ray; hence, he was sent for cardiac computed tomography (CT). CT revealed a large TAA of size 5.5 $\mathrm{cm}$ involving the aortic arch (Fig. 1). Surgical treatment for the TAA was recommended but he refused surgery due to concerns about the risk of surgery.

He had been hospitalized twice earlier, 5 months and 3 months ago, and was provided conservative treatment for nausea, intermittent chest discomfort, and vague dysphagia. At the time of earlier hospitalization, blood chemistry showed hyponatremia that was attributed to the long-term use of diuretics, and his symptoms had rapidly improved with fluid therapy, antacids and gastrointestinal motility drugs; thus, he was discharged without further evaluation.

However, he was admitted again with the same symptoms. A detailed medical history was taken to identify the accurate cause of dysphagia. Symptoms were gradual in onset and were aggravated on swallowing solid food. At times, vomiting accompanied by mild chest discomfort occurred shortly after swallowing. The symptoms indicated occult esophageal disease. Therefore, upper gastrointestinal endoscopy, barium esophagram, and chest CT were performed to identify the cause of dysphagia.

Upper gastrointestinal endoscopy showed prominent impressions with a compromised lumen in the mid and lower esophagus. 


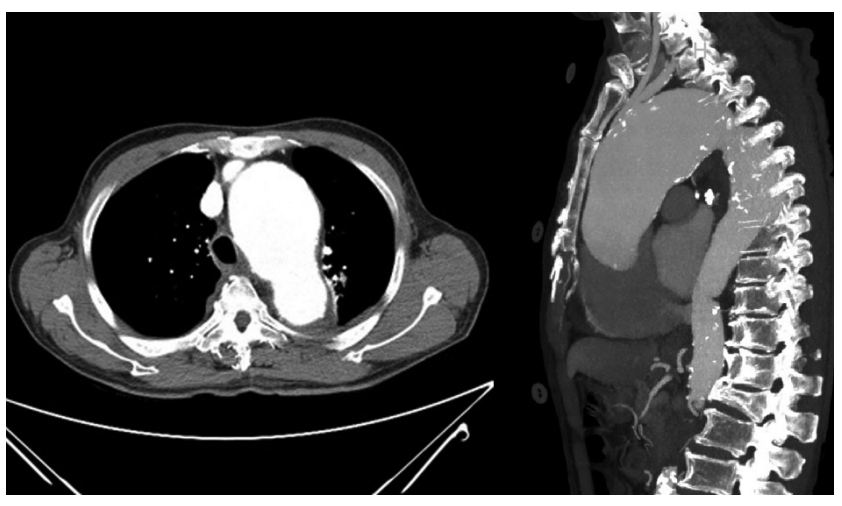

Fig. 1. Cardiac computed tomography revealed a large thoracic aortic aneurysm of size $5.5 \mathrm{~cm}$ involving the aortic arch.
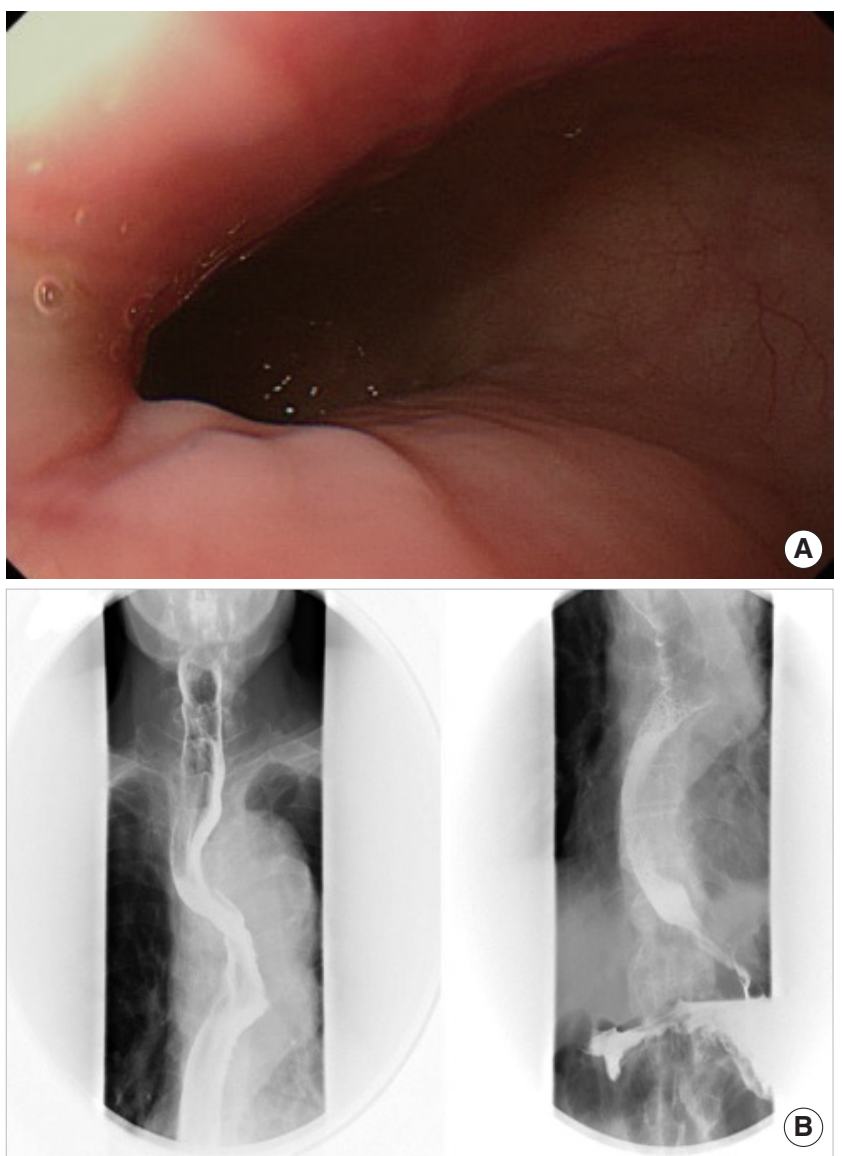

Fig. 2. (A) Upper gastrointestinal endoscopy showed prominent impressions with a compromised lumen in the mid and lower esophagus. (B) Barium esophagram showed tortuosity and compressions of the esophagus at the levels of the aortic arch and lower half of the thoracic descending aorta.

The barium esophagram showed tortuosity and compressions of the esophagus at the levels of the aortic arch and lower half of the thoracic descending aorta (Fig. 2). A prominent dilation of the TAA (maximum $7 \mathrm{~cm}$ in size) and compression of the esophagus
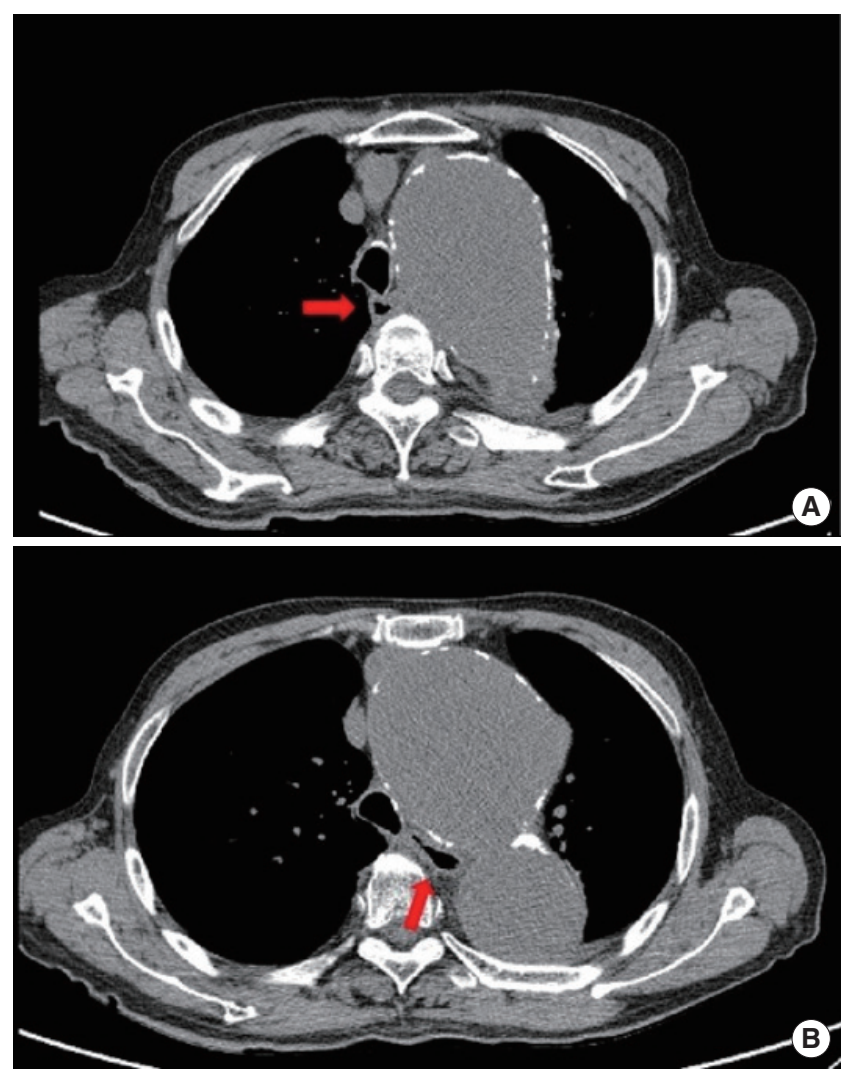

Fig. 3. (A, B) Chest computed tomography without contrast agent showed thoracic aortic aneurysm that was more expanded for 2 years (maximum $7 \mathrm{~cm}$ in size). Arrows indicate deviated and compressed esophagus.

were seen on chest CT without contrast agent (Fig. 3).

Given these test results, dysphagia aortica was diagnosed. Surgical correction of the thoracic aneurysm was recommended but was refused by the patient. Dietary modifications such as liquid diet, chewing small amounts of soft diet were attempted for treatment. His symptoms gradually improved and he was discharged after 2 weeks of hospitalization. He is currently doing well with dietary modification.

\section{DISCUSSION}

The term dysphagia aortica was first described by Pape [2] in 1932 to describe the difficulties in swallowing caused by an aortic aneurysm as presented here. Dysphagia aortica is a rare type of dysphagia caused by an extrinsic mechanical compression of esophagus. The esophagus normally crosses the aorta in the lower third of the posterior mediastinum. At this level, the diseased aorta with dilation or distortion pushes and compresses the esophagus against the cardiac chambers, resulting in dysphagia. Dyspha- 
gia arising from compression of the esophagus by the aortic arch is relatively rare [3].

Patients with dysphagia aortica usually present with dysphagia for solids but not for liquids, unless the lumen is severely compromised. Dysphagia to liquids is related more to disorders of esophageal motility. However, as the compression progresses, dysphagia for liquids follows [4].

In the presented case, our patient was admitted repeatedly due to vomiting and vague swallowing difficulty. However, an early diagnosis for dysphagia aortica could not be made because his symptoms were intermittent and not severe. We misdiagnosed hyponatremia as the cause of his symptoms, due to the history of long-term use of diuretics for heart failure.

The diagnosis of dysphagia aortica is difficult. Most cases with TAA are identified incidentally and have no symptoms related to an aneurysm. Symptoms occur when the size of the aneurysm increases rapidly or complications such as aortic dissection or rupture develop. Until date, several cases of dysphagia aortica have been reported, but the exact incidence has not been established. It is rare and even when large, does not necessarily result in dysphagia. According to previous reports, the incidence of dysphagia aortica is higher in women and in old patients as well as in those with short stature, hypertension, and kyphosis [5,6].

A barium esophagram can suggest the diagnosis based on the appearance of a partial obstruction and a pulsatile movement, synchronous with aortic pulsation. Esophageal manometry might reveal a localized high-pressure band with abnormal motility [5]. Endoscopy might show extrinsic compression of the esophagus, and pulsations might be seen. Chest CT, the most important diagnostic modality, can provide findings supporting the diagnosis of dysphagia aortica by revealing a TAA and a displaced esophagus. However, there is no gold standard diagnostic procedure for dysphagia aortica. The radiographic findings are often inconclusive because a dilated, tortuous thoracic aorta is frequently encountered in elderly patients with dysphagia. False negative results are common in barium swallow studies and vascular pulsation commonly appears during endoscopy of the normal esophagus as well. In addition, the classical manometric features suggestive of dysphagia aortica can also be seen in normal subjects [7]. The diagnosis should be based on the patient's symptoms such as progressive intolerance to solids with concomitant weight loss and comprehensive evaluation through various tests including the imaging modalities mentioned above.
Management of dysphagia aortica is limited mostly to dietary modification such as liquefaction of food. The treatment of underlying heart failure and hypertension has been advised in cases of mild dysphagia. Occasionally, if the dysphagia is severe and if the patient is eligible for a surgical procedure, separation of the esophagus from the aorta and repair of the aneurysm might be undertaken.

Thoracic endovascular aortic repair (TEVAR) is an alternative to open surgery because it is less invasive and has shown low morbidity rate in TAA treatment. Two contemporary case reports of TEVAR in treating TAA with dysphagia showed considerable symptom relief and the safety of the procedure [8,9]. Although there is a concern about esophageal erosion resulting in aortoesophageal fistula due to TEVAR [10,11], it might be considered for patients who cannot be treated surgically.

Coelho-Prabhu and Baron [12] reported esophageal stenting in dysphagia aortica for the first time in 2009. Their patient's symptoms rapidly improved, and the patient was able to tolerate a full diet, despite remaining at high risk due to TAA. Since then however, esophageal stenting alone in dysphagia aortica has never been reported in the literature. There might be a risk of stent distortion by the pulsatile aorta and a risk of aortic rupture due to the aortic wall erosion induced by the self-expansible property of the stent. Further evidence for the esophageal stenting is needed in dysphagia aortica.

Our patient had a high surgical risk and more importantly, he refused surgical treatment. TEVAR was also contraindicated because his TAA involved the branches of aortic arch. However, his dysphagia was not severe, and significantly improved with dietary modification alone. If the patient's symptoms are further aggravated by the gradual expansion of the aortic aneurysm, we will inevitably have to consider esophageal stenting or percutaneous endoscopic gastrostomy.

In conclusion, dysphagia aortica is a rare cause of dysphagia and can be improved by conservative and surgical or interventional treatments. It is important to remember that a TAA can cause dysphagia, which can prevent delayed diagnosis and catastrophic complications.

\section{REFERENCES}

1. Taylor CW, Sinha A, Nightingale JM. Dysphagia and thoracoabdominal aneurysm. Postgrad Med J 2001;77:257-8. 
2. Pape R. Uber einen abnormen verlauf ('tiefe Rechtslage') der mesa aotitischen aorta descendens. Fortschr Roetgenstr 1932;46:257-69.

3. Di Bisceglie AM, Segal I, Mirwis J. Benign extrinsic oesophageal dysphagia: case reports. S Afr Med J 1985;67:219-21.

4. Abdul Haziz SR, Bickle I, Chong VH. Dysphagia aortica: a rare cause of dysphagia. BMJ Case Rep 2015;2015:bcr2015211726.

5. Wilkinson JM, Euinton HA, Smith LF, Bull MJ, Thorpe JA. Diagnostic dilemmas in dysphagia aortica. Eur J Cardiothorac Surg 1997;11:222-7.

6. Ponce J, Gaspar E, Del Val A, Garrigues V, Sancho-Tello MJ, Carrasquer J. Dysphagia of vascular origin. Rev Esp Enferm Dig 1991;80:53-6.

7. Kim JH, Jang SW, Kim DB, Lee HJ, Kim JG, Kwon BJ, et al. A patient with dysphagia due to an aortic aneurysm. Korean Circ J 2009;39:258-60.

8. Kische $\mathrm{S}$, Werner $\mathrm{D}$, Ince $\mathrm{H}$. A neglected symptom of contained aortic laceration: dysphagia aortica successfully treated by endovascular stent- grafting. Catheter Cardiovasc Interv 2012;80:1052-5.

9. Siddiqui J, Hughes F. Dysphagia due to thoracic aortic aneurysm, relieved by thoracic endovascular aneurysm repair: a case report and review of the literature. BMJ Case Rep 2011;2011:bcr0920114793.

10. Xi EP, Zhu J, Zhu SB, Zhang Y. Secondary aortoesophageal fistula after thoracic aortic aneurysm endovascular repair: literature review and new insights regarding the hypothesized mechanisms. Int J Clin Exp Med 2014; 7:3244-52.

11. Yavuz S, Kanko M, Ciftci E, Parlar H, Agirbas H, Berki T. Aortoesophageal fistula secondary to thoracic endovascular aortic repair of a descending aortic aneurysm rupture. Heart Surg Forum 2011;14:E249-51.

12. Coelho-Prabhu N, Baron TH. Dysphagia and weight loss in an elderly person: dysphagia aortica. Gastroenterology 2009;137:e1-2. 